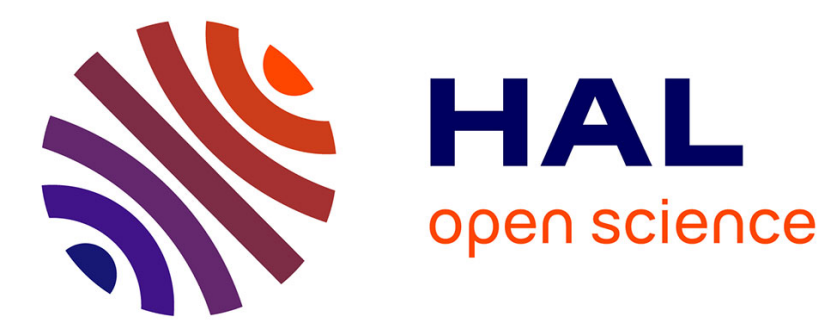

\title{
Mixture Effects in Contaminant Reemission
}

Thomas Tondu, Emilie Vanhove, Jean François Roussel, Delphine Faye

\section{To cite this version:}

Thomas Tondu, Emilie Vanhove, Jean François Roussel, Delphine Faye. Mixture Effects in Contaminant Reemission. Journal of Spacecraft and Rockets, 2016, 53 (6), p. 1172-1177. 10.2514/1.A33507 . hal-01488136

\section{HAL Id: hal-01488136 https://hal.science/hal-01488136}

Submitted on 13 Mar 2017

HAL is a multi-disciplinary open access archive for the deposit and dissemination of scientific research documents, whether they are published or not. The documents may come from teaching and research institutions in France or abroad, or from public or private research centers.
L'archive ouverte pluridisciplinaire HAL, est destinée au dépôt et à la diffusion de documents scientifiques de niveau recherche, publiés ou non, émanant des établissements d'enseignement et de recherche français ou étrangers, des laboratoires publics ou privés. 


\title{
Mixture Effects in Contaminant Reemission
}

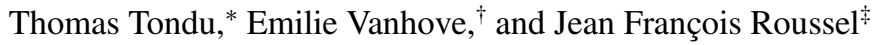 \\ ONERA-The French Aerospace Lab, 31055 Toulouse, France \\ and \\ Delphine Faye $\mathrm{s}^{\S}$ \\ Centre National d'Études Spatiales, 31401 Toulouse, France
}

DOI: $10.2514 / 1 . \mathrm{A} 33507$

\begin{abstract}
The deposit of a pure contaminant or of contaminant mixtures may exhibit complex behavior. This impacts the possible in-flight reemission of these deposits as well as the interpretation of thermogravimetric analyses. These effects were studied, first on theoretical grounds, and then experimentally on controlled mixtures of pure contaminants or uncontrolled mixtures stemming from a real material outgassing. Several effects were clearly identified, such as phase segregation and various mixture behaviors, corresponding to quick or slow diffusion of the contaminants within the mixture.
\end{abstract}

\section{Nomenclature}

\begin{tabular}{|c|c|c|}
\hline$c_{1}$ & & $\begin{array}{l}\text { contaminant } 1 \text { concentration in fraction of } \\
\text { monolayer }\end{array}$ \\
\hline$c_{\text {bulk }, i}$ & & $\begin{array}{l}\text { bulk concentration of contaminant } i \text { in fraction of a } \\
\text { monolayer }\end{array}$ \\
\hline$c_{\mathrm{dep}, i}$ & & $\begin{array}{l}\text { ation of contaminant } i \text { in the deposit in } \\
\text { of a monolayer }\end{array}$ \\
\hline$c_{\text {surf }, i}$ & 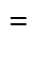 & ation of contaminant $i$ in fraction of \\
\hline & & efficient in monolayers per time unit \\
\hline & & \\
\hline $\mathrm{d} m_{i} / \mathrm{d} t$ & & $\begin{array}{l}\text { of contaminant } i \text { in the mixture in } \\
\text { time unit }\end{array}$ \\
\hline $\mathrm{d} x_{\mathrm{dep}} / \mathrm{d} t$ & $=$ & $\begin{array}{l}\text { speed of the surface of the evaporating deposit in } \\
\text { monolayers per time unit }\end{array}$ \\
\hline$m_{2}$ & $=$ & contaminant 2 mass in monolayers \\
\hline$v_{\text {pure }, i}$ & $=$ & $\begin{array}{l}\text { ation rate of contaminant } i \text { in a pure system in } \\
\text { ayers per time unit }\end{array}$ \\
\hline$x$ & & from the deposit surface in monolayers \\
\hline$x_{\mathrm{dep}}$ & & thickness of the evaporating deposit in monolayers \\
\hline$x_{p}$ & $=$ & $\begin{array}{l}\text { thickness of contaminant beyond which the } \\
\text { contaminant concentration is its bulk concentration }\end{array}$ \\
\hline
\end{tabular}

\section{Introduction}

$\mathbf{I}^{\mathrm{r}}$ N-FLIGHT spacecraft contamination is driven by four main phenomena: contaminant generation (outgassing, propulsion, etc.), transport, reemission, and fixation by space radiation [1, 2]. No correct in-flight contamination assessment can be produced if one of these phenomena is neglected. This paper focuses on the reemission phenomenon and, more specifically, on the interactions between the contaminants deposited on a surface. Contamination is composed of a high number of contaminants generated by a large number of materials, each outgassing several chemical species. Within a

Received 5 November 2015; revision received 30 June 2016; accepted for publication 6 July 2016; published online 8 September 2016. Copyright (C) 2016 by the American Institute of Aeronautics and Astronautics, Inc. All rights reserved. Copies of this paper may be made for personal and internal use, on condition that the copier pay the per-copy fee to the Copyright Clearance Center (CCC). All requests for copying and permission to reprint should be submitted to CCC at www.copyright.com; employ the ISSN 00224650 (print) or 1533-6794 (online) to initiate your request.

*Research Engineer, Space Environment Department.

†Research Engineer, Space Environment Department; emilie.vanhove@ onera.fr.

${ }^{*}$ Head of Space Environment Department, Space Environment Department; Jean-Francois.Roussel@onera.fr.

${ }^{\S}$ Material and Contamination Engineer, Quality Insurance; delphine.faye@ cnes.fr. deposit, the interactions between contaminants affecting reemission are not yet totally established.

Evaporation of a pure contaminant can be simply described. Thick film deposits have an order 0 evaporation rate at a given temperature. It is given by the Langmuir law. Temperature dependence for pure contaminant evaporation is given by the Clausius-Clapeyron law. As a result, the evaporation rate of a pure contaminant is almost an Arrhenius law characterized by an activation energy and a preexponential factor $[1,2]$. We previously showed that this simple description can be insufficient to describe reemission of a deposit generated by vacuum deposition, for two main reasons. The deposit can evolve into clusters, which exhibit a different vacuumcontaminant interface than a film. Furthermore, contaminants can be either amorphous or crystallized, with different evaporation parameters. Vacuum deposition tends to form amorphous deposits at very low temperature. This amorphous deposit can undergo crystallization. This transformation has a complex kinetic driven both by nucleation and crystal growth.

Contaminant mixtures can condense on cold surfaces in a crystallized or amorphous deposit. The possibility for two different contaminant molecules to crystallize together in a single phase is almost null. On the other hand, amorphous homogeneous mixtures can develop when molecular mobility is low in the deposit. The formation of several phases and molecular mobility in the deposit are of high importance in the contaminant reemission process.

Thermogravimetric analysis (TGA), performed by measuring the evaporation rate of a deposit that is heated at constant rate, is a classical characterization method for reemission kinetics. TGA can be applied to deposits generated by real space material outgassing $[3,4]$. For all the aforementioned reasons, the interpretation of these TGA experiments is not easy. In this paper, we focus on identifying the physical processes that govern evaporation of a mixture of contaminants during a TGA experiment. In the first part, we treat theoretical aspects of contaminant evaporation in binary systems. In the second part, we present evaporation experiments of controlled mixtures of two and three contaminants. The experimental results are discussed and modeled. In the third part, we present TGA experiments of outgassing products of the adhesive Scotchweld EC2216. These TGAs are monitored by mass spectrometry.

\section{Theoretical Aspects of Mixture Evaporation}

We consider a homogenous system composed of two contaminants (contaminants 1 and 2). At the experiment temperature, each contaminant $i$ is characterized by its evaporation rate in a pure system $v_{\text {pure }, i}$. For simplification, we express the rates in monolayers per time unit. We also assume that one monolayer of each contaminant corresponds to the same amount of contaminants.

Only contaminants with access to vacuum can evaporate. We assume that each contaminant has the same affinity for other 
contaminants as for itself. With these assumptions, the evaporation rate of each contaminant is proportional to its surface concentration (expressed as a fraction of a monolayer, hence a dimensionless number smaller than 1) and to its own evaporation rate in a pure system:

$$
\frac{\mathrm{d} m_{i}}{\mathrm{~d} t}=-c_{\text {surf }, i} \cdot v_{\text {pure }, i}
$$

In the absence of diffusion, surface concentrations evolve, leading to an enrichment in low-volatility contaminant and impoverishment in high-volatility contaminants.

The contaminant $i$ concentration variation in the last monolayer is described by Eq. (2):

$$
\frac{\mathrm{d} c_{\text {surf }, i}}{\mathrm{~d} t}=\frac{\mathrm{d} m_{i}}{\mathrm{~d} t}-c_{\text {bulk }, i} \sum_{j} \frac{\mathrm{d} m_{j}}{\mathrm{~d} t}
$$

where $\mathrm{d} m_{i} / \mathrm{d} t$ is the evaporation of contaminant $I$, and $-c_{\text {bulk }, i}$. $\mathrm{d} m_{i} / \mathrm{d} t$ is the uncovering of contaminant $i$ molecules due to the evaporation of contaminant $j$.

In the steady state, $\mathrm{d} c_{\text {surf }, i} / \mathrm{d} t=0$. As a result, the flux of each contaminant is proportional to its bulk concentration:

$$
\frac{\mathrm{d} m_{i}}{\mathrm{~d} t}=c_{\mathrm{bulk}, i} \frac{\mathrm{d} m}{\mathrm{~d} t}
$$

and for a binary mixture:

$$
\frac{\mathrm{d} m_{1}}{\mathrm{~d} t}=-c_{\text {bulk }, 1} \frac{v_{\text {pure }, 1} v_{\text {pure }, 2}}{v_{\text {pure }, 1} c_{\text {bulk }, 2}+v_{\text {pure }, 2} c_{\text {bulk }, 1}}
$$

which allows to deduce the total evaporation rate for a binary mixture:

$$
\frac{\mathrm{d} m}{\mathrm{~d} t}=-\frac{v_{\text {pure }, 1} v_{\text {pure }, 2}}{v_{\text {pure }, 1} c_{\text {bulk }, 2}+v_{\text {pure }, 2} c_{\text {bulk }, 1}}
$$

At a given temperature, evaporation rates of different contaminants can vary over several orders of magnitude, and the probability of similar evaporation rates for two contaminants is very low. For very different evaporation rates, the total evaporation rate is driven by the evaporation of the contaminant with the smallest volatility, which evaporates as if it were pure:

$$
\text { if } v_{\text {pure }, 1} c_{\text {bulk }, 2} \gg v_{\text {pure }, 2} c_{\text {bulk }, 1} \quad \frac{\mathrm{d} m}{\mathrm{~d} t}=-\frac{v_{\text {pure }, 2}}{c_{\text {bulk }, 2}}
$$

This phenomenon is illustrated by Fig. 1, where a TGA of a homogeneous deposit containing $75 \%$ of contaminant 1 and $25 \%$ of contaminant 2 is simulated using Eq. (2). The evaporation rates of each contaminant in the mixture, and as pure contaminant, are presented. In this simulation, contaminant 1 is much more volatile

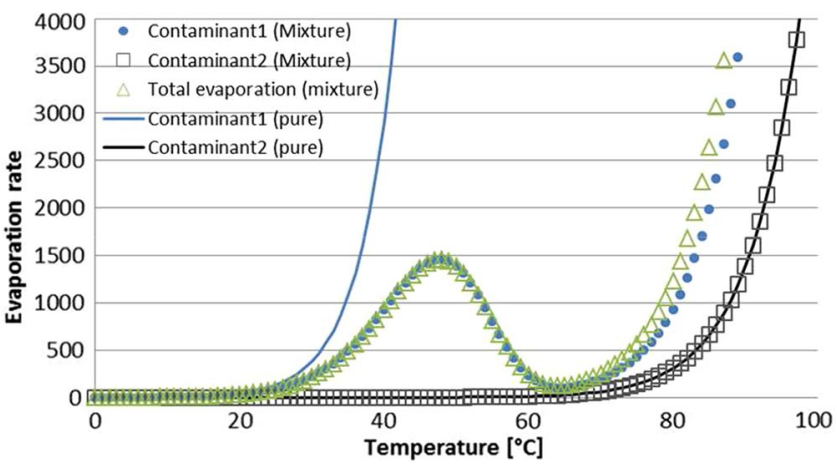

Fig. 1 TGA simulation, homogeneous deposit containing $75 \%$ of contaminant 1. than contaminant 2. Emission of contaminant 1 is observed around $50^{\circ} \mathrm{C}$. This emission corresponds to the transition from a homogeneous deposit to a deposit impoverished in contaminant 1 in the surface (contaminants that have access to vacuum evaporate). After that, contaminants are emitted in proportion to their bulk concentrations (three times more contaminant 1 than 2). Contaminant 2 evaporates as if it were pure. The main TGA peak is 4 times the contaminant 2 peak. This is also equivalent to a temperature shift.

We now consider the opposite case with a very rapid diffusion within the contaminant deposit. This means that surface composition always equals bulk composition. We consider the case of a volatile contaminant (contaminant 1) in solution with a nonvolatile contaminant (contaminant 2). The contaminant 1 concentration (and correlated evaporation rate) is governed by the following differential equation:

$$
\frac{\mathrm{d} c_{1}}{\mathrm{~d} t}=\frac{\mathrm{d}}{\mathrm{d} t}\left(\frac{m_{1}}{m_{1}+m_{2}}\right)=-\frac{\nu_{\text {pure }, 1}}{m_{2}} c_{1}\left(1-c_{1}\right)^{2}
$$

If $c_{1}$ is small, the concentration decreases exponentially. Figure 2 presents the shapes of TGA peaks for the same amount of volatile contaminant diluted at three concentrations in a nonvolatile contaminant. The higher the dilution is, the higher the emission temperature is. This simulation was performed with an activation energy of $100 \mathrm{~kJ} / \mathrm{mol}$ for the contaminant evaporation. It leads to a temperature shift of less than $20^{\circ} \mathrm{C}$ in peak position, when the pure contaminant gets diluted to $10 \%$.

The rapid diffusion, however, raises questions because it supposes a high molecular mobility. In this case, two contaminants at solid state would tend to crystallize separately.

On the other hand, mobility can be small without being null. In this case, the volatile contaminant evaporation rate follows a classical $t^{-1 / 2}$ law, whereas the second contaminant remains condensed.

We showed previously that, in the absence of diffusion, evaporation of the high-volatility contaminant is controlled by the emission of the low-volatility contaminant. In the case of slow diffusion, the decrease of the concentration gradient close to the surface, caused by diffusion, can be counterbalanced by the lowvolatility contaminant evaporation. In the referential following the moving deposit surface, which is in translation because of the deposit thickness decrease, the high-volatility contaminant profile $c_{1}(x, t)$ is governed by the following Lagrangian diffusion equation:

$$
\frac{\mathrm{d} c_{1}}{\mathrm{~d} t}=\frac{\mathrm{d} x_{\mathrm{dep}}}{\mathrm{d} t} \frac{\mathrm{d} c_{1}}{\mathrm{~d} x}+D \frac{\mathrm{d}^{2} c_{1}}{\mathrm{~d} x^{2}}
$$

In this equation, the first term, $\mathrm{d} x / \mathrm{d} t\left(\mathrm{~d} c_{1} / \mathrm{d} x\right)$, is due to the displacement of the deposit surface caused by evaporation, and the second term, $D \mathrm{~d}^{2} c_{1} / \mathrm{d} x^{2}$, is due to diffusion. In the steady state, $\mathrm{d} c_{1} / \mathrm{d} t=0$, and for a binary system,

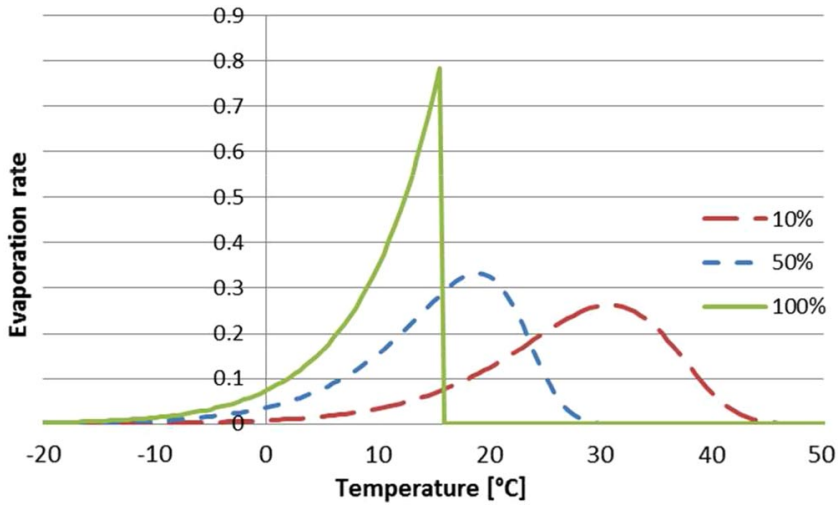

Fig. 2 TGA simulation, contaminant diffusing rapidly in a nonvolatile contaminant, volatile contaminant initial concentrations: 10, 20, and $100 \%$. 


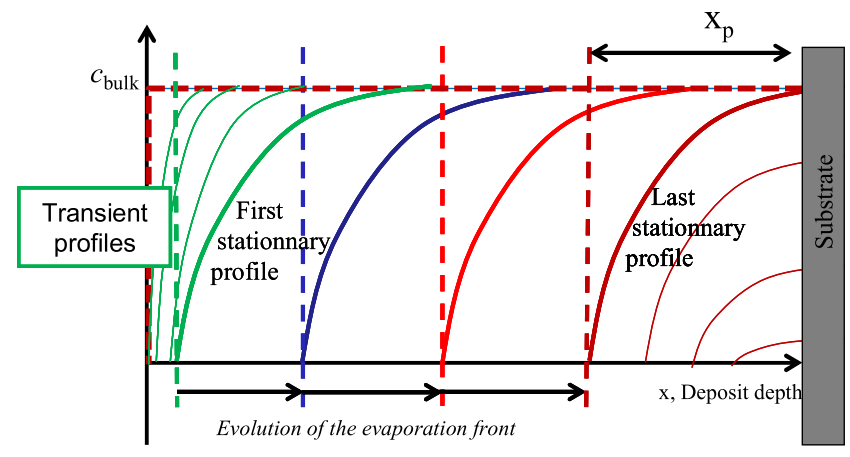

Fig. 3 Evolution of the concentration of the volatile contaminant during the evaporation.

$$
\frac{\mathrm{d}}{\mathrm{d} t}\left(c_{\mathrm{dep}, 1}\right)=0 \quad \frac{\mathrm{d} m_{1}}{\mathrm{~d} t}=c_{\mathrm{dep}, 1} \frac{\mathrm{d} m}{\mathrm{~d} t}
$$

Using Eq. (1):

$$
\frac{\mathrm{d} m}{\mathrm{~d} t}=-\frac{v_{\text {pure }, 1} v_{\text {pure }, 2}}{v_{\text {pure }, 1} c_{\mathrm{dep}, 2}+v_{\text {pure }, 2} c_{\mathrm{dep} 1}}
$$

$$
\begin{aligned}
& \text { if } v_{\text {pure }, 1} c_{\mathrm{dep}, 2} \gg v_{\mathrm{pure}, 2} c_{\mathrm{dep}, 1} \\
& \frac{\mathrm{d} m}{\mathrm{~d} t}=-\frac{v_{\mathrm{pure}, 2}}{c_{\mathrm{dep}, 2}}=-\frac{\mathrm{d} x_{\mathrm{dep}}}{\mathrm{d} t} \\
& \frac{\mathrm{d}^{2} c_{1}}{\mathrm{~d} x^{2}}=-\frac{v_{\mathrm{pure}, 2}}{D c_{\mathrm{dep}, 2}} \frac{\mathrm{d} c_{1}}{\mathrm{~d} x} \\
& c_{1}=c_{\mathrm{surf}, 1} \exp \frac{-v_{\text {pure }, 2}}{D\left(1-c_{\mathrm{dep}, 1}\right)} x+c_{\mathrm{dep}, 1}\left(1-\exp \frac{-v_{\text {pure }, 2}}{D\left(1-c_{\mathrm{dep}, 1}\right)} x\right) \\
& c_{1} \approx c_{\mathrm{dep}, 1}\left(1-\exp \frac{-v_{\text {pure }, 2}}{D\left(1-c_{\mathrm{dep}, 1}\right)} x\right)
\end{aligned}
$$

This stationary solution only rigorously exists for an infinitely thick deposit. In practice, the deposit concentration is only modified from its bulk concentration over a thickness $x_{p}$. Figure 3 shows this thickness and the stationary phase (thick profiles) of the evaporation, between the first stationary profile and the last stationary profile. Thin lines show the initial and final transient phases.

The evaporation regime indeed remains stationary as long as the deposit keeps larger than $x_{p}$, which is given by

$$
x_{p}=\left(1-c_{\mathrm{dep}, 1}\right) \cdot D / v_{\text {pure }, 2}
$$

Actually, the stationary assumption remains valid as long as the contaminant concentration at the bottom of the deposit remains equal to the initial bulk concentration.

The main difference between no diffusion and slow diffusion is that the stationary regime is reached in the absence of diffusion when volatile contaminant of the first contaminant monolayer get evaporated, whereas it is reached when the stationary profile has established on several monolayers in the case of slow diffusion.

Furthermore, in TGA conditions, $x_{p}$ variations depend on relative activation energies of $D$ and of the low-volatility contaminant evaporation rate. If $x_{p}$ remains constant with temperature (same activation energies), the TGA is similar to Fig. 1, except for the fact that the first peak area is proportional to $x_{p}\left(x_{p}=1\right.$ in the absence of diffusion). If $x_{p}$ increases with temperature, the emission of the highvolatility contaminant increases more rapidly than the emission of the low-volatility contaminant and inversely. The stationary regime stops when $x_{p}=x_{\mathrm{dep}}$.

\section{Experiments}

Characterization of contaminant evaporation was performed in ONERA-The French Aerospace Lab's COPHOS facility (from

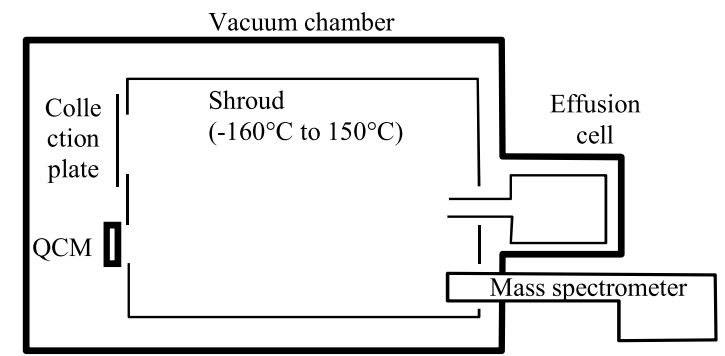

Fig. 4 Schematic view of the COPHOS facility.

"contamination photon synergy") dedicated to simulating contamination processes, such as outgassing, reemission kinetics, and photofixation (see Fig. 4).

The setup is based on an effusion cell $\left(20-200^{\circ} \mathrm{C}\right)$ for molecular beam generation and on four independently temperature-controlled Quartz crystal microbalances (QCMs) (Maxtek $6 \mathrm{MHz}$ ) for deposit monitoring. The QCMs are in direct view of the effusion cell. This assembly is surrounded by a shroud that can be either cooled down to cryogenic temperature to stick any contaminant and prevent indirect contamination on QCMs or heated up above effusion cell temperature to reevaporate any contaminant. In this second hot configuration, contaminants collected by the QCMs have been condensed and reevaporated several times on the shroud. This second configuration allows the use of a mass spectrometer for analysis of the outgassed contaminants or of contaminants reemitted by QCM heating.

We also used temperature-controlled contaminant collectors for contaminant reemission characterization by TGA. In this configuration, plates of typically $10 \mathrm{~cm}^{2}$ have been contaminated and decontaminated by heating at constant temperature rate. The evaporated contaminants could be thus characterized by mass spectrometry.

QCM frequencies depend on temperature. This dependence has been characterized and subtracted from TGA measurements.

We generated a controlled mixture of up to three contaminants. The simultaneous deposition of contaminants was managed by introducing up to three crucibles containing pure contaminants in the effusion cell. The crucibles are closed by plates with a hole. The hole sizes were adapted to tune each contaminant flux. It is very difficult to find molecular contaminants with similar evaporation rates. The tuning of the hole sizes allowed generating similar contaminant fluxes even for volatility ratios between contaminants as high as 1 to 1000. We chose bisphenol A (BPA), octadecan-3-one (OD), and triphenylmethane (TPM) as pure contaminants. These contaminants have been chosen for their compatibility toward the generation of a similar flux with the three crucible strategy. They are also expected to have significantly different mass spectrometry responses.

\section{Results and Discussion}

\section{A. Pure Contaminant}

Each contaminant was first separately characterized by measuring the outgassing rate (open crucible conditions) as a function of temperature. Reevaporation rates were also measured, by applying temperature steps (the temperature is kept constant, and the resulting constant reevaporation rate is measured for each temperature step) or temperature ramps (classical TGA for each pure contaminant) to the QCMs.

Two series of evaporation rates can be observed, depending on whether the deposit is crystallized or amorphous. Figure 5 presents the case of TPM evaporation rate measurement. Here, both TGAs and two of the step measurements are characteristic of amorphous deposit, whereas the six other step measurements exhibit the characteristic of crystallized deposits. Crystallized deposit at low temperature was only observed after heating the deposit above $10^{\circ} \mathrm{C}$.

\section{B. Binary Mixture}

Several TGAs of the BPA/TPM mixture were performed. Figure 6 presents a typical experiment. 


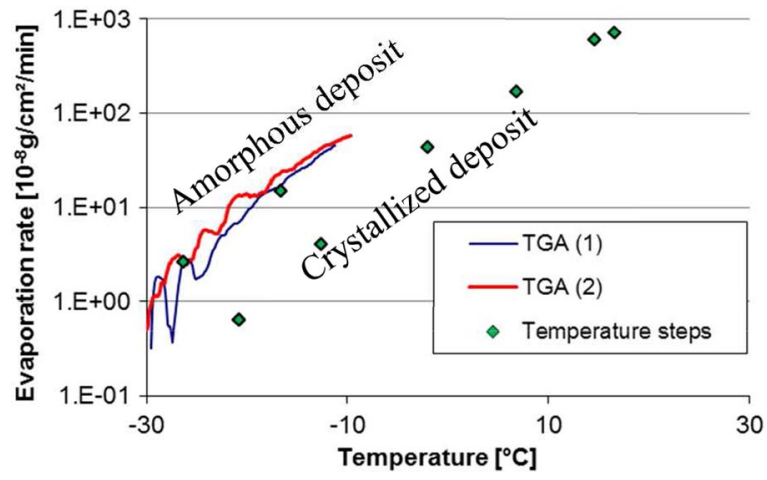

Fig. 5 TPM evaporation rate measurement for two TGAs and for temperature steps.

The TGA of the BPA/TPM mixture is compared to the TGA of pure contaminants and to a simulation. Three peaks appear; the first one is consistent in position with pure TPM, but it only corresponds to a fraction of the TPM present in the deposit. The last peak is consistent with the evaporation of BPA (position and area). The intermediate peak at approximately $15^{\circ} \mathrm{C}$ is attributed to TPM (for mass conservation reason) and is observed when BPA evaporation starts. Extra experiments were performed to help interpreting this experiment and are represented in Figs. $\underline{7}$ and $\underline{8}$.

Figure 7 compares two compositions (BPA $60 \%-\mathrm{TPM} 40 \%$ and BPA 35\%-TPM 65\%) and two amounts (350 and $45010^{-8} \mathrm{~g} / \mathrm{cm}^{2}$ ) of the mixture BPA/TPM that correspond to a comparable amount (210 and $16010^{-8} \mathrm{~g} / \mathrm{cm}^{2}$ ) of BPA, whereas Fig. 8 compares two compositions (BPA 60\%-TPM 40\% and BPA 35\%-TPM 65\%) and two amounts (350 and $29010^{-8} \mathrm{~g} / \mathrm{cm}^{2}$ ) of the mixture BPA/TPM that correspond to a comparable amount $\left(140\right.$ and $\left.19010^{-8} \mathrm{~g} / \mathrm{cm}^{2}\right)$ of TPM. As expected, for the same amount of BPA in the mixture Fig. 7 , the BPA peak remains unchanged. More surprisingly, the first

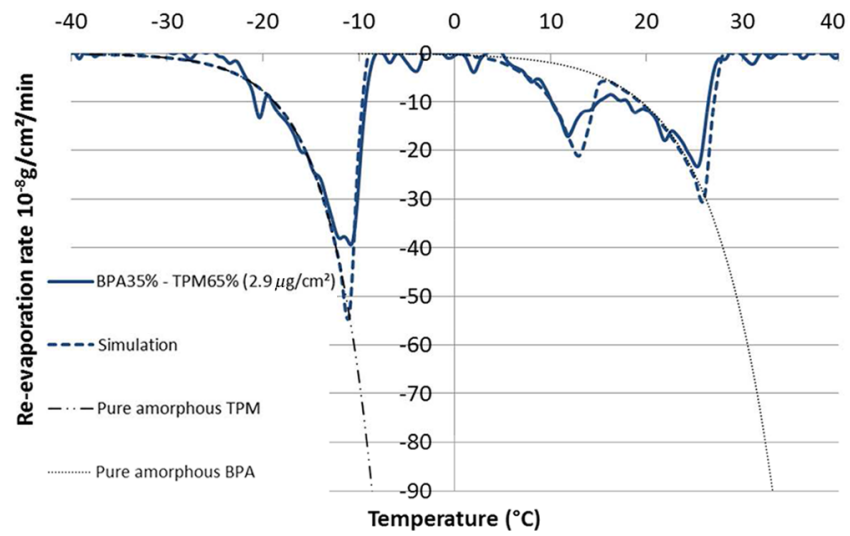

Fig. 6 TGA of $29010^{-8} \mathrm{~g} / \mathrm{cm}^{2}$ of $40 \% \mathrm{BPA}$ and $60 \% \mathrm{TPM}$.

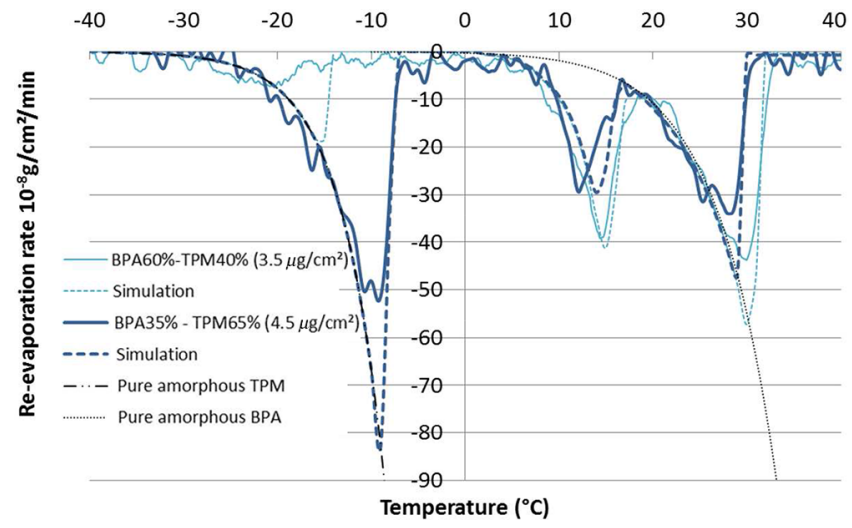

Fig. 7 TGA of BPA/TPM mixtures: comparable amount of BPA.

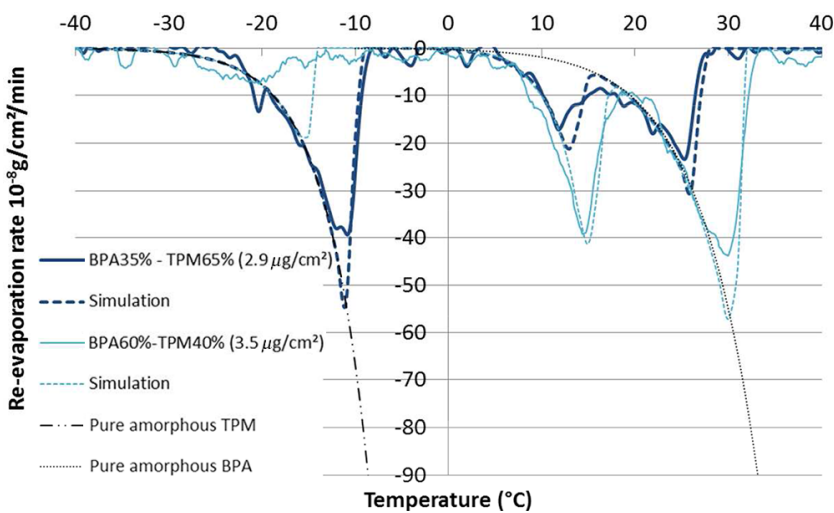

Fig. 8 TGA of BPA/TPM mixtures: comparable amount of TPM.

TPM peak clearly depends on the TPM concentration and not on the total amount of TPM. Indeed, for a comparable amount of TPM Fig. 8 , the first peak is much smaller with the $40 \%$ TMP mixture than with the 65\% TPM mixture. The TGAs of Figs. 6-8 can thus be interpreted by the formation of two phases: a pure $\overline{\mathrm{TP}} \overline{\mathrm{M}}$ phase and a homogeneous TPM/BPA phase. A total of six experiments with the TPM/BPA mixture showed that the concentration of TPM in the mixture after the total evaporation of the first peak can be assessed to be $40 \%$. This concentration is considered to be the limit of solubility of TPM in BPA (in solid phase). The dissolved TPM is released when the BPA evaporation starts. Figures 7 and 8 show that this emission can be extremely sharp and is incompatible with a classical TGA interpretation. Finally, these TGAs where simulated (Figs. ㅁ-무) assuming slow diffusion [Eq. (8)] and $c_{\text {surf.TPM }} \ll 1$. As it can be seen in these figures, simulations fit the experimental curves very well.

The evaporation of this phase was also studied by measuring the evaporation rate at a constant temperature of a $40 \%$ TPM mixture rapidly heated up to $4.25^{\circ} \mathrm{C}$ after its deposition. Figure 9 presents the evaporation rate at constant temperature as a function of time. The $4.25^{\circ} \mathrm{C}$ temperature is above the first peak evaporation (cf. Fig. 6), and so this evaporation must be interpreted as the evaporation of the homogeneous BPA/TPM mixture. A constant evaporation rate can be observed during the first 45 min of evaporation. Then, the evaporation rate slowly decreases down to the pure BPA evaporation rate. The initial evaporation rate indicates that TPM evaporation cannot be explained by rapid diffusion. Otherwise, evaporation rate would follow an order 1 law. On the other hand, the fact that the final deposit is compatible with pure BPA indicates that some diffusion occurs.

These first $45 \mathrm{~min}$ can indeed be explained by the steady-state evaporation rate and concentration profile given in Eq. (10). The end of steady-state evaporation is reached when the moving steady concentration profile reaches the substrate (Fig. 3). Then, the evaporation rate continuously decreases down to the BPA evaporation rate, which is reached when all TPM is evaporated.

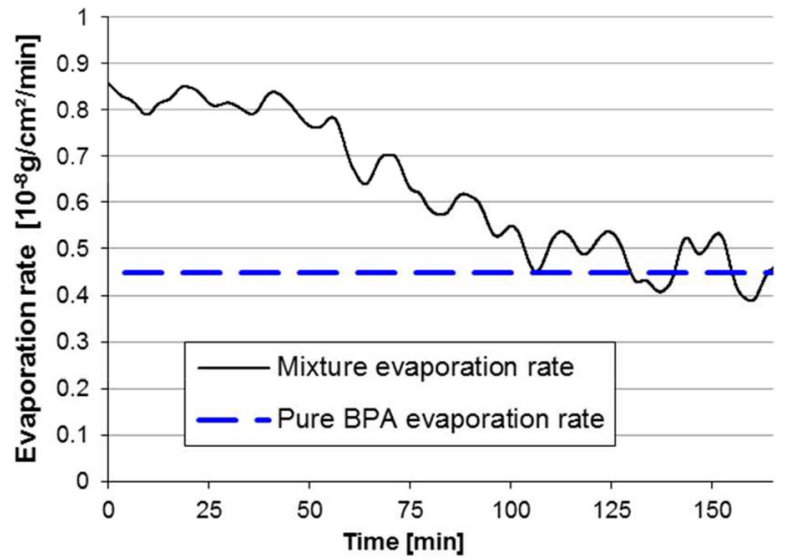

Fig. 9 Evaporation rate of a $60 \%$ TPM-40\% BPA mixture at constant temperature $4.25^{\circ} \mathrm{C}$. 


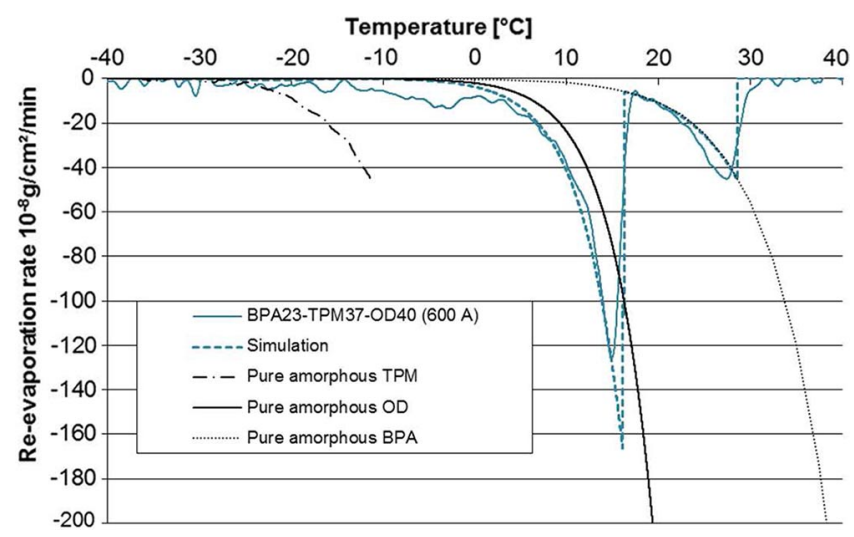

Fig. 10 TGA of contaminant mixtures and pure contaminants.

From this experiment, and using Eq. (11), a diffusion coefficient of TPM in BPA at $4.25^{\circ} \mathrm{C}$ close to $10^{-16} \overline{\mathrm{cm}}^{2} / \mathrm{s}$ was deduced. This is a very low diffusion coefficient but is sufficient to allow TPM diffusion within thin TPM/BPA films.

As a conclusion on Sec. IV.B, we can say that the TPM/BPA TGA experiments are quite well explained by phase segregation due to limited solubility of TPM in BPA. The pure TPM phase evaporates like a pure TPM thin film. After evaporation of this TPM phase, the mixture evaporates like a homogeneous deposit with slow diffusion, as described previously. The very sharp second TPM peak observed in Figs. $\underline{6}-\underline{8}$ can be explained by a high diffusion activation energy.

\section{Ternary Mixture}

A third contaminant, OD, was added to the binary BPA/TPM mixture. Figure 10 presents the influence of adjunction of OD (40\% in the deposit) to a BPA 40\%-TPM $60 \%$ mixture.

In the ternary mixture, the first TPM peak disappears, whereas another peak quite similar to OD evaporation, but higher in intensity (or shifted to lower temperatures), appears. For mass conservation reasons, the OD-like peak must also contain TPM evaporation. This explains the fact that evaporation rate is higher than that for pure OD. This observation is compatible with the formation of a TPM/OD phase and a BPA phase. The evaporation of the TPM/OD phase is compatible with the evaporation in absence of diffusion (or very limited diffusion) described previously. Simulation, based on Eq. (2), thus satisfyingly fits the experimental curves.

For confirmation of these hypotheses, a similar experiment was then performed using a collecting plate in the hot shroud configuration (reevaporation of all contaminants reaching the shroud). The deposit was collected on the plate and then reevaporated at a constant temperature rate (TGA). The reevaporated contaminants were simultaneously monitored using a cold QCM $\left(-75^{\circ} \mathrm{C}\right)$ and mass spectrometry. For each contaminant, a mass, specific to this contaminant, was monitored during the plate heating ramp at $2^{\circ} \mathrm{C} / \mathrm{min}$.

Figure 11 presents the deposition rate on the QCM compared to the mass deposition rate deduced from the mass spectrometry signal. An excellent correlation between QCM and mass spectrometry measurements is observed. Looking now at each mass, it clearly appears that the TPM is mainly emitted simultaneously with the OD. Nevertheless, a fraction of TPM is released slightly before the emission OD, whereas a second fraction is released at the beginning of the BPA evaporation. OD and BPA are almost emitted like pure contaminants. The TGA and mass spectrometry experiment thus clearly indicates that the deposit demixes into two phases, composed of OD/TPM and TPM/BPA mixtures, respectively. For each phase, the emission of TPM is limited by its slow diffusion.

TThis is a bit surprising because this phase should only cover a fraction of the total surface, leading to an evaporation rate lower than that for a pure TPM deposit.

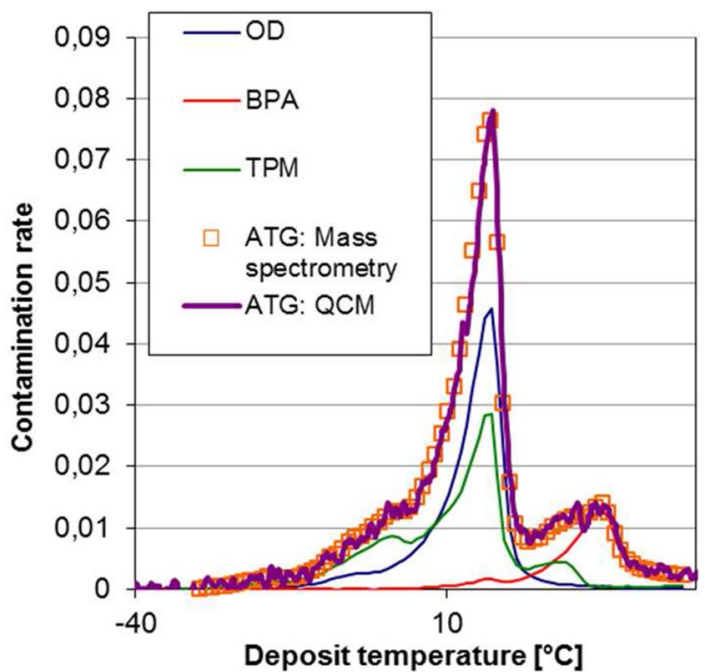

Fig. 11 TPM/OD/BPA mixture TGA monitored by mass spectrometry and cold QCM.

\section{Outgassing Products from a Real Material}

Finally, experiments were conducted on the deposit obtained from real material outgassing (Scotchweld EC2216 adhesive). We made four successive outgassing steps $\left(75,100,125\right.$, and $\left.150^{\circ} \mathrm{C}\right)$, each one lasting approximately $20 \mathrm{~h}$. During each of the four steps, the deposit was collected on cold plates $\left(-75^{\circ} \mathrm{C}\right)$ and then reevaporated during a TGA experiment at $2^{\circ} \mathrm{C} / \mathrm{min}$. Contaminant reevaporation was monitored in the hot shroud configuration using a cold QCM and a mass spectrometer. Contaminant collection after outgassing at $75^{\circ} \mathrm{C}$ was small, and mass spectrometry data were not treated. The following figures compare TGA and mass spectrometry measurements for the three next outgassing steps. Figure 12 shows the TGA performed after each temperature step $\left(100,125\right.$, and $\left.150^{\circ} \mathrm{C}\right)$.

The difficulty that we have highlighted, with controlled contaminant mixtures, is that one TGA peak is not systematically characteristic of one contaminant (TPM with OD), and one mass spectrometry mass can be characteristic of several contaminants [5]. Furthermore, mass spectrometry response is not directly correlated to the contaminant mass flux. We nevertheless tried to interpret these data using mass spectrometry measurements performed during the TGAs.

Figure 13 presents the mass signals for mass 76 a.m.u (atomic mass unit). and mass 47 a.m.u. during the three TGA. The signals of these two masses are proportional whatever the temperature (except small differences at $-50^{\circ} \mathrm{C}$ ). This clearly shows that these two masses correspond to the same contaminant, outgassed at the three temperature steps. The comparison to Fig. 12 shows that the 76/47 doublet emission peaks are highly correlated with TGA peaks (total

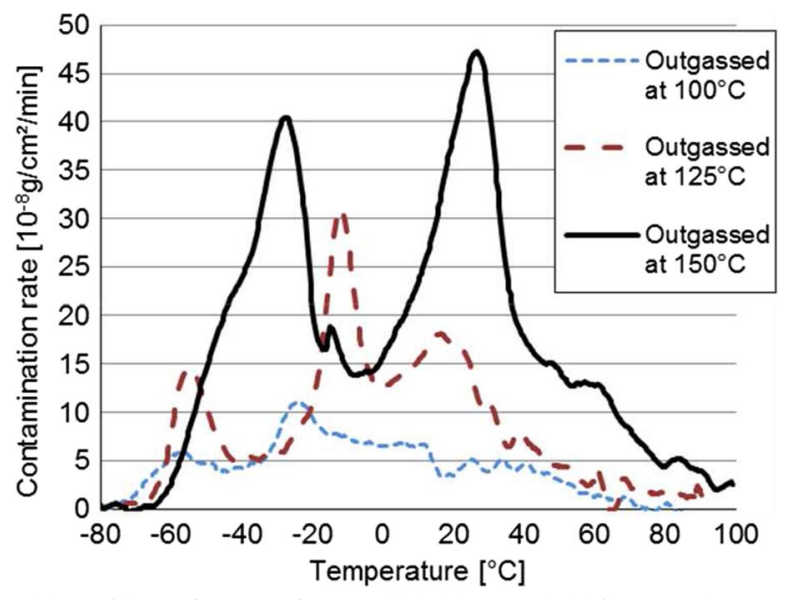

Fig. 12 TGA performed after the 100,125 , and $150^{\circ} \mathrm{C}$ outgassing steps. 


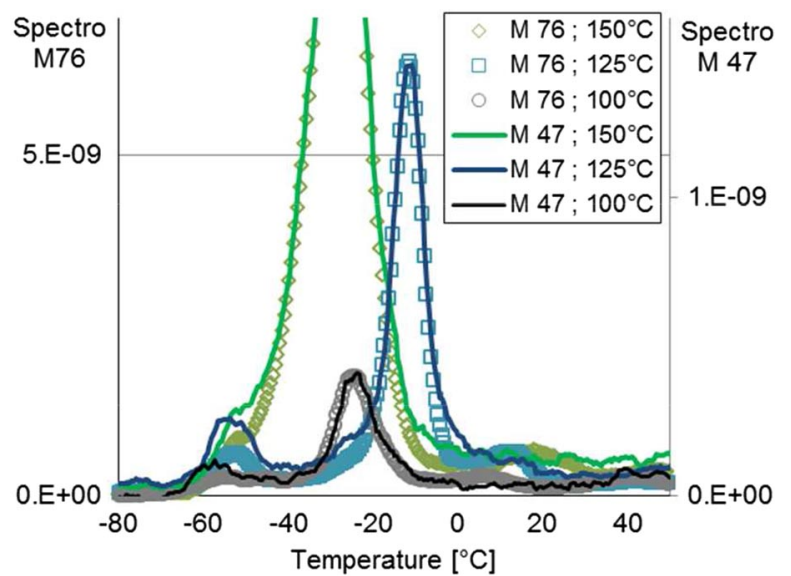

Fig. 13 Masses 47 and 76 during TGAs performed after the 100, 125, and $150^{\circ} \mathrm{C}$ outgassing steps.

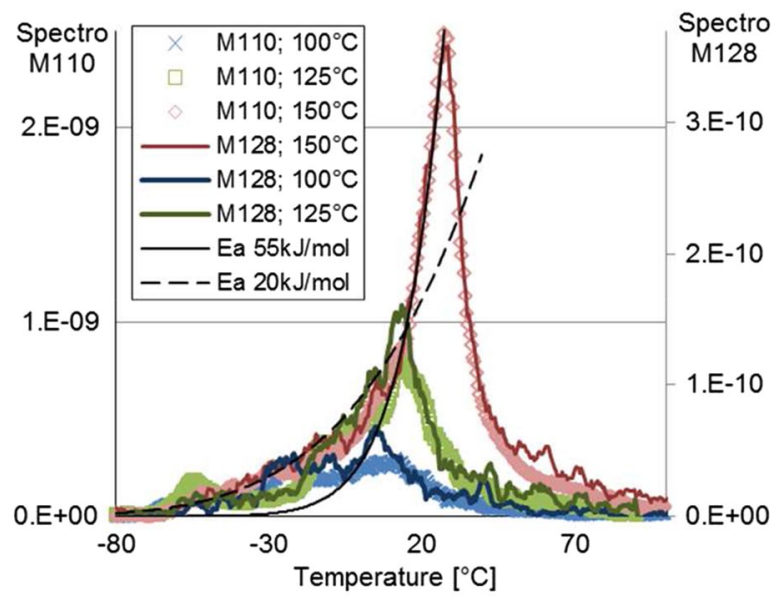

Fig. 14 Masses 110 and 128 during the three TGAs.

reevaporation rate). This means that the contaminant with characteristic peaks 76 and 47 is a main contaminant.

The same observation is made for the doublet 110/118 (Fig. 14).

However, the behavior of these two contaminants is different. The $76 / 47$ contaminant peak is sharp, but its position depends on the studied TGA, hence the mixture composition. The outgassing of this contaminant is thus probably controlled by other contaminants in a mixed phase. On the other hand, the contaminant $110 / 128$ has a wide peak, but with unchanged emission law for the three TGAs. This is typical of a pure contaminant behavior (or of the dominant contaminant of a mixed phase).

Figure 15 finally presents the second reemission peak of the TGA performed after the $150^{\circ} \mathrm{C}$ temperature step (peak shown in Fig. 12). The behavior of four different contaminants, monitored during this TGA by mass spectrometry is also shown. The intensity of the reemission peak of each contaminant is very difficult to assess because relative abundances of ions in the mass spectrum obtained for a mixture do not necessarily reflect the proportions of the compounds in the mixture. Because we are interested in the position of these peaks but not their intensity, they are normalized so that the maximum intensity of the peak is 1 . Masses 79 and 110 present doublet behavior (79/108 and 110/128) and are supposed to be representative of two contaminants. Masses 239 and 109 are characteristic of two different outgassing products, identified by gas chromatography (unpublished). It can thus be concluded that this TGA peak actually corresponds to at least four different contaminants, which are emitted almost simultaneously. The reevaporation of the contaminants is thus probably limited here by diffusion (no or slow diffusion).

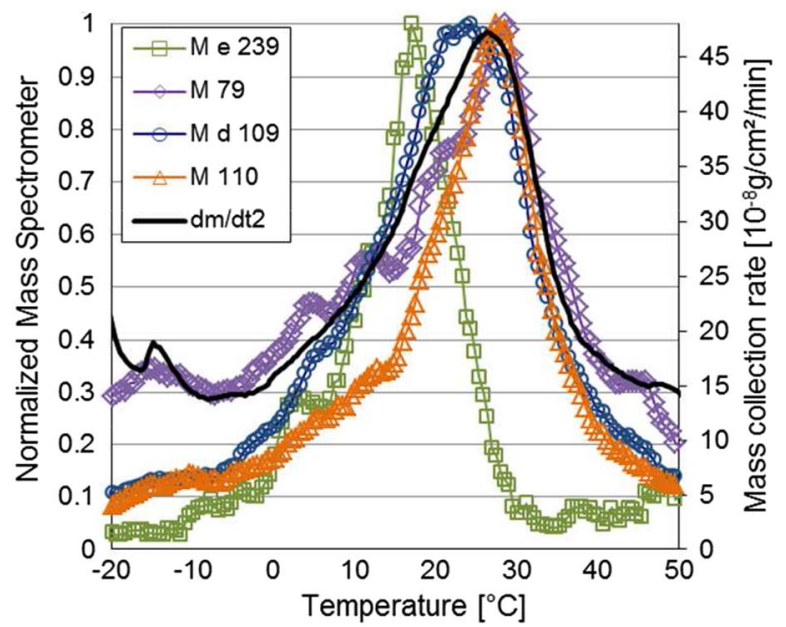

Fig. 15 TGA performed after the $150^{\circ} \mathrm{C}$ outgassing step: total mass and various mass signals.

\section{Conclusions}

Contamination generated by spacecraft material outgassing can be analyzed by thermogravimetric analysis (TGA), but its interpretation is not easy. The formation of several phases of different composition is highly probable. In each phase, diffusion is often slow, so that during TGA, emission of the most volatile contaminant is limited by diffusion and is triggered by the evaporation of the less-volatile contaminant. This has been clearly demonstrated with controlled mixture of selected materials. In the case of deposit generated by real material outgassing, species separation was only partially achieved thanks to mass spectrometry. Behaviors typical of those identified in the controlled mixtures were identified.

The next steps of these activities shall consist of actually separating the species present in complex deposits coming from the outgassing of real materials. When studying more materials, an important question will be whether the nontrivial mixing effects unraveled here are dominant or, on the contrary, might be neglected in many real situations.

\section{Acknowledgments}

The results obtained at ONERA-The French Aerospace Lab were funded by the Centre National d'Études Spatiales (CNES) Research and Technology Program, for which the ONERA authors are very grateful to the CNES.

\section{References}

[1] Tribble, A. C., Fundamentals of Contamination Control, SPIE Press, Bellingham, Washinghton, D.C., 2000, pp. 26-38, 40-42.

[2] Roussel, J.-F., Tondu, T., Paulmier, T., Faye, D., Van Eesbeek, M., and Rampini, R., "Progress on the Physical Approach to Molecular Contamination Modeling," Journal of Spacecraft and Rockets, Vol. 48, No. 2, 2011, pp. 246-255. doi: $10.2514 / 1.49490$

[3] "Kinetic Outgassing of Materials for Space," European Space Agency, Requirements and Standards Division, Rept. ECSS-Q-TM-70-52A, Noordwijk, The Netherlands, 2009.

[4] "Standard Test Method for Contamination Outgassing Characteristics of Spacecraft Materials," American Society for Testing and Materials Rept. ASTM E1559-09, West Conshohocken, PA, 2009.

[5] Anderson, M. S., "Mass Spectrometry of Spacecraft Contamination Using Direct Analysis in Real-Time Ion Source," Journal of Spacecraft and Rockets, Vol. 51, No. 1, 2014, pp. 374-378. doi: 10.2514/1.A32613 